
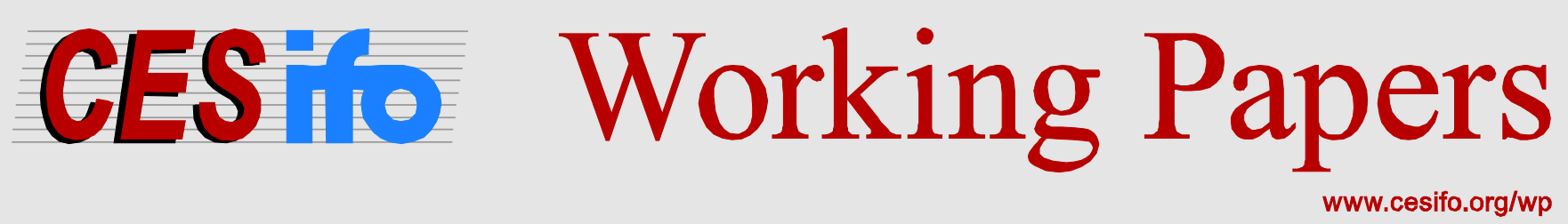

\title{
Anticipated Consumption and its Impact on Capital Accumulation and Growth: 'Forward-Looking' vs. 'Backward-Looking' Consumption Reference
}

\author{
Goncalo Monteiro \\ Stephen J. Turnovsky
}

CESIFO WORKING PAPER NO. 4536

CATEgory 6: Fiscal Policy, Macroeconomics AND Growth

DECEMBER 2013

An electronic version of the paper may be downloaded

- from the SSRN website:

- from the RePEc website:

- from the CESifo website:

WwW.SSRN.com

www.RePEc.org

www.CESifo-group.org/wp

\section{CESifo}




\title{
Anticipated Consumption and its Impact on Capital Accumulation and Growth: 'Forward-Looking' vs. 'Backward-Looking' Consumption Reference
}

\begin{abstract}
It has long been argued that the anticipations of some future event will impact current consumption and well-being in advance of the occurrence of the event itself. This paper introduces this idea of anticipated pleasure into the Ramsey growth model, by assuming that in addition to his own current consumption, an agent's current utility depends upon a reference consumption level that is based on expected future consumption. Two alternative specifications of the anticipated future consumption levels are considered: an external index and an internal index. We analyze the macrodynamic equilibrium, comparing it to both the standard Ramsey model, and the more familiar parallel model of habit formation. We establish a number of theoretical propositions characterizing the impact of the anticipated consumption reference on the transitional dynamics and long-run equilibrium, supplementing these with extensive numerical simulations.
\end{abstract}

JEL-Code: D900, E200.

Keywords: anticipated consumption, capital accumulation.

\author{
Goncalo Monteiro \\ State University of New York at Buffalo \\ NY / USA \\ gm43@buffalo.edu
}

\author{
Stephen J. Turnovsky \\ University of Washington \\ Seattle WA 98195 / USA \\ sturn@u.washington.edu
}

August 2013

This paper has benefited from presentation to the 18th SCE conference on Computing in Economics and Finance held in Vancouver in July 2013. We also wish to thank Ben Keefer for helpful comments. Stephen Turnovsky's research was supported in part by the Castor and Van Voorhis endowments at the University of Washington. This support is gratefully acknowledged. 


\section{Introduction}

The standard Ramsey growth model assumes that individuals' utility functions at any point of time depend upon only contemporaneous variables, specifically their own current consumption and in many cases leisure (labor supply). The limitations of this time-separable specification of utility have become increasingly recognized, and there is a growing literature in which current utility depends not only on current consumption, but also on past consumption levels, which collectively may provide a benchmark against which current consumption can be assessed. These so-called models of habit-formation were first introduced by Ryder and Heal (1973), and over the years have been refined and applied to a variety of issues with varying degrees of success. These applications include: asset pricing (Constantinides, 1990, Campbell and Cochrane, 1999), consumption behavior (Osborn, 1988, Fearson and Constantinides, 1991, Dynan, 2000), short-run macroeconomic stabilization (Ljundqvist and Uhlig, 2000, Fuhrer, 2000), exchange rate behavior (Mansoorian, 1988) and economic growth (Carroll, Overland, and Weil, 1997, 2000, Alvarez-Cuadrado, Monteiro, and Turnovsky, 2004, Alonso-Carrera, Caballé, and Raurich, 2005, Turnovsky and Monteiro, 2007).

While past consumption levels may form a natural benchmark against which current consumption, and therefore current welfare, may be assessed, it is also plausible that anticipations of future consumption levels may be an equally relevant consideration. There are at least two distinct reasons one can advance in support of this view. The first is an old one, associated with the idea of the "pleasures of anticipations", and dates back to Jevons (1871) and Marshall (1891). In this vein, the phrase "I am looking forward to that (event)" is one of the most widely used expressions, and it is clearly articulating the notion that the anticipation of the future event being referred to is increasing the individual's current well-being, in advance of the event itself. The first author to investigate more formally the impact of anticipated consumption on current utility was Loewenstein (1987). The focus of his study was to relate the pleasure associated with the process of anticipating future consumption (or its pain in some circumstances) to the standard practice of time discounting of future consumption. He showed how the utility from anticipation may cause individuals to delay positive consumption experiences, thereby enabling them to prolong the pleasures of their 
anticipatory experience.

The second justification is as a potential benchmark for comparative purposes. It seems reasonable that forward-looking agents, in assessing their current well-being, may be equally concerned with comparing their present consumption to their expected future consumption profile, just as they may relate it to their past consumption habits. Indeed, this comparison was recognized some time ago by Elster and Loewenstein (1992) who referred to it as the "contrast effect"1 More recently, the notion that the reference point should be based on expectations has been emphasized by Kőszegi and Rabin $(2006,2007)$ in their seminal work on consumption and gain-loss utility.

Despite the importance of anticipated future consumption as a determinant of current utility, there is almost no literature examining its macroeconomic consequences. One study by Kuznitz, Kandel, and Fos (2008) examines the implications of anticipated consumption on portfolio choice. Another more recent study by Faria and McAdam (2013) discusses its consequences for equilibrium in a monetary economy, and its implications for the effectiveness of monetary policy. Both papers find that the introduction of anticipated future consumption can have significant consequences. The objective of the present paper is to examine the consequences that anticipated future consumption may have for capital accumulation and economic growth.

The relationship of current well-being to agents' experiences at different points in time is a complex one, involving a range of psychological aspects with which economists have generally limited familiarity. In the case of past experiences, there are past observations (past consumption), that together, can provide concrete sources of information regarding agents' consumption patterns. Most economic applications that use this information to form some measure of habit, do so by either treating the previous period's consumption level as the reference point (e.g. Fuhrer, 2000, AlonsoCarrera et al., 2005) or more commonly, by constructing the habit as some exponentially declining weighted average of all past consumption levels (e.g. Ryder and Heal, 1973, Carroll et al. 1997, 2000, Ljundqvist and Uhlig, 2000, Alvarez-Cuadrado et al. 2004, Turnovsky and Monteiro, 2007). However, research by psychologists suggest that the way agents process past information is much

${ }^{1}$ Elster and Loewenstein (1992, p225) write: “Anticipated experiences also affect current well-being via the contrast effect, by serving as a point of comparison against which current consumption is measured." 
more complex than that, in that they may be heavily influenced by extreme events, or circumstances that prevailed at formative stages of their lives, such as when they began employment. ${ }^{2}$ How well this more complex behavior associated with cognitive science is captured by the conventional economic model of habits, with its exponentially declining weights, is questionable.

Matters are even more complex with regard to anticipations, in that, without having observations on which to anchor the anticipations, they are inherently uncertain and the psychological aspects assume even greater importance. One issue stressed in the psychological literature involves anxiety and anticipatory emotions; see Caplin and Leahy (2001) and Köszegi (2010). As these authors note, these attributes introduce the potential for time inconsistent decision making behavior. While we acknowledge these aspects, we will represent anticipated future consumption in a way parallel to those traditionally employed to describe habits, namely as a discounted sum of future consumptions, which, under the assumption of perfect foresight that we shall also invoke, are known with certainty. We therefore abstract from the issues relating to anxiety and time inconsistency. From this standpoint, our focus is primarily on the comparison between two alternative approaches to formulating reference consumption levels - specifically past consumption versus (expected) future consumption - and we are therefore not attempting to deal with the more subtle psychological aspects that we have alluded to.

As we discuss in more detail below, the "habit formation" literature adopts two alternative specifications of the benchmark consumption level. The first is as an external index based on past economy-wide consumption levels, which the individual, being atomistic, takes as given. Since in equilibrium this index reflects the collective behavior of all individuals, it serves as an externality, leading to distortions in consumption. ${ }^{3}$ The alternative approach is as an internal index, based on the agent's own past consumption levels, and which he chooses as part of his optimal consumption plan.

We suggest that precisely the same distinction is applicable to a consumption reference index

\footnotetext{
${ }^{2}$ Rubin, Weltzer, and Nebes (1986) were the first to document how memories of events experienced between the ages of 10 and 30 are more vividly stored and easily recalled, a phenomenon referred to in the psychological literature as the "reminiscence bump".

${ }^{3}$ Dupor and Liu (2003) emphasize the distortionary aspect in the case where aggregate economy-wide consumption is introduced contemporaneously into the individual's utility function. Turnovsky and Monteiro (2007) undertake a similar task when the consumption reference point is based on the discounted sum of past economy-wide consumption levels.
} 
based on anticipated future consumption levels. There is however, one significant difference, which arises when the index is internal. Whereas in the "habits" context the index is adapted from past behavior, so that its initial value is a historically given initial condition, the initial value of the internal index based on future anticipations is unconstrained in this way. Instead, its choice is part of the agent's constrained intertemporal optimization problem. ${ }^{4}$

Using this framework we shall derive and analyze the macrodynamic equilibrium in an economy where, in addition to his own current consumption, an agent's current utility depends upon a reference consumption level that is based on a discounted measure of expected future consumptions. We begin with the case where the reference pertains to expected economy-wide consumption, which the individual is too small to influence, and therefore takes as given. This measure operates as an externality and is therefore immune to the types of issues discussed in the psychological literature. In addition to comparing this formulation to the standard Ramsey model, we also contrast it with the conventional model of habit formation, where the reference point is based on past behavior. We then proceed to the case where the reference index is formed internally, being based on the agent's own anticipated consumption path, and carry out a similar comparison. Insofar as possible, we proceed analytically, although to gain further insight we supplement the formal analysis with the numerical simulations of a plausibly calibrated aggregate growth model.

Several key results emerge from our combination of formal analysis and numerical simulations. First, under the commonly adopted assumption of inelastic labor supply, the presence of a consumption reference point in utility - whether it be external or internal, backward-looking, or forward-looking - has no long-run effects on the levels of output, capital, or consumption. Hence, the steady state is identical to that of the conventional Ramsey model. In contrast, the structure of the consumption reference point has potentially a substantial impact on the nature of the transitional dynamics. Thus, the introduction of an external forward-looking consumption reference leads to an equilibrium macrodynamic system characterized by a one-dimensional stable locus. This compares with the more familiar backward-looking external consumption reference case, where the transitional

\footnotetext{
${ }^{4}$ In terms of the terminology introduced by Köszegi and Rabin (2006), the reference point is chosen as an endogenously determined expectation, rather than as the status quo.
} 
adjustment is two dimensional. This difference arises because the anticipated reference stock can jump on impact in response to new information as it is received, whereas the habit reference is constrained to adjust gradually over time. Moreover, if expectations about future consumption decrease the marginal utility of current consumption, the introduction of an external reference consumption point reduces the incentive to accumulate capital and consequently decreases the rate of convergence.

If labor is supplied elastically, both external and internal consumption reference points will generally have real productive effects. In the external case the effect operates via its impact on the marginal rate of substitution between consumption and labor, in which case it has no effect if preferences are represented by the widely employed constant elasticity utility function. In contrast, for this utility function an internally generated consumption reference which enhances utility has a positive effect on the long-run level of employment, capital, output and consumption. This occurs whether the reference point is forward-looking or backward-looking. In addition, the long-run effect of an increase in the weight on near-future consumption in the anticipated future consumption reference level is contractionary, thus, contrasting with the habit-based consumption reference level, which is expansionary.

Finally, the numerical simulations illustrate how introducing an internal reference consumption level, has markedly different effects on the transitional dynamics, depending upon whether it is forward-looking or backward-looking. These differences apply not only to what happens on impact, but also to the nature of the entire subsequent transitional path.

The remainder of the paper is structured as follows. Following this introduction we discuss some of the issues pertaining to the specification of a forward-looking benchmark versus a backward-looking benchmark in more detail. Section 3 sets out the equilibrium for the external index, while Section 4 does so for the internal case. Numerical calibrations and simulations are conducted and discussed in Section 5. Section 6 briefly discusses some of the long-run consequences of endogenizing labor supply, while Section 7 concludes. Since the details of the backward-looking reference are well known, there is no need to develop that case in detail. Instead, 
we simply summarize the corresponding equilibrium, for convenience, in the Appendix.

\section{Utility and backward-looking versus forward-looking reference consumption levels}

The conventional assumption that an agent's welfare depends on only his own current consumption, $C$ say, independent of others, has long been criticized; see e.g. Veblen (1899), and Duesenberry (1949). The initial approach to countering this objection was to modify the usual welfare integral to

$$
\int_{0}^{\infty} U(C, R) e^{-\beta t} d t
$$

where $R$ denotes the consumption level of some reference group. In the earliest developments this was taken to be the contemporaneous consumption level of some peer group - possibly the entire economy - which the individual is too small to influence. In this case, $R$ operates as a pure consumption externality that may be either positive or negative, depending upon how the reference group impacts upon the individual's well-being. If $U_{R}<0$ a ceteris paribus increase in the consumption level of the reference group reduces the agent's utility due to jealousy, while if $U_{R}>0$ the individual is altruistic and his utility increases with the general consumption level; see Dupor and Liu (2003). If $R$ raises the marginal utility of consumption, this characterizes "keeping up with the Joneses" and has generated a voluminous literature; see e.g. Abel (1990), Gali (1994). ${ }^{5}$

This formulation maintains the property that utility is "time-separable", meaning that the agent's well-being at time $t$, say, depends only upon what is occurring at that same instant. An alternative approach is to assume that the consumption reference level involves consumption at other points of time, thereby rendering the utility function to be "time-nonseparable". The existing solutions are associated with backward-looking measures of reference consumption levels; that is, the reference consumption level depends upon the time path of past consumption levels. In this approach, the reference point may be constructed from the past consumption levels (rather than the

\footnotetext{
${ }^{5}$ Dupor and Liu (2003) also identify what they call "running away from the Jones" as the case where an increase in the $R$ reduces the marginal utility of consumption.
} 
present) of the external reference group, or alternatively it may be based on the agent's own past consumption levels. In the former case it is said to be "externally generated" and the agent described as being "outward-looking", while in the latter case it is "internally generated" and the agent characterized as being "inward-looking"; see e.g. Carroll, Overland, and Weil (1997, 2000), Alvarez-Cuadrado, Monteiro, and Turnovsky (2004), Alonso-Carrera, Caballé, and Raurich. (2005), among others.

As noted, this backward-looking specification was first introduced by Ryder and Heal (1973), where intertemporal utility was specified to be of the form:

$$
\int_{0}^{\infty} U(C, H) e^{-\beta t} d t \quad U_{C}>0, U_{H}<0
$$

and

$$
H(t)=\kappa \int_{-\infty}^{t} C(\tau) e^{-\kappa(\tau-t)} d \tau
$$

so that the consumption habit level at time $t$, denoted by $H(t)$, is an exponentially declining weighted average of past consumption. ${ }^{6}$ Differentiating $(2 \mathrm{~b})$ with respect to time yields

$$
\dot{H}(t)=\kappa(C(t)-H(t)) \quad \kappa>0
$$

One critical assumption introduced by Ryder and Heal, and prevalent throughout the subsequent literature, is that an increase in the consumption habit, with current consumption unchanged, will reduce the agent's utility, $U_{H}<0$. For the widely adopted constant elasticity utility function, $U=(1 / \gamma)\left(\mathrm{CH}^{-\theta}\right)^{\gamma}$, this implies that habits, serving as a reference, and current consumption are Edgeworth complements in the sense that $U_{C H}>0$, if and only if the intertemporal elasticity of substitution, $(1-\gamma)^{-1}<1$, as empirical evidence overwhelmingly suggests. ${ }^{7}$ However, $^{2}$ drawing on the comprehensive empirical study of Houthakker and Taylor (1966) and Taylor and

\footnotetext{
${ }^{6}$ The parameter $\kappa$ characterizes the weight given to past consumption levels; the smaller $\kappa$, the slower the weight declines over time.

${ }^{7}$ Two consumption goods, $X$ and $Y$, are said to be Edgeworth complements if their cross derivative $U_{X Y}(X, Y)>0$; they are Edgeworth substitutes if $U_{X Y}(X, Y)<0$.
} 
Houthakker (2010), to the extent that the consumption good is durable, one can interpret $H$ as the (depreciated) accumulation of past consumption flows, in which case it is plausible for $U_{H}>0$. Indeed, a characteristic of any durable good, such as an automobile, is that it will provide benefits extending over multiple time periods, not just during the period in which it was purchased. ${ }^{8}$ As a result, the stock of past consumption, $H$, reduces the marginal utility of current consumption, in which case durability and current consumption are Edgeworth substitutes, $U_{C H}<0$. The stock of past consumption, $H$, can thus be interpreted as reflecting both a negative "reference effect" and a positive "durability effect", with the overall signs of $U_{H}, U_{C H}$ depending upon which is dominant .

While Ryder and Heal interpreted $H$ in terms of internally generated habits, they also acknowledged that it may represent the evolution of external habits. More recently, Carroll et al. (1997) and Alvarez-Cuadrado et al. (2004) consider both formulations in their analysis of growth and find that they generate very similar dynamic time paths for the aggregate variables. In this context, $U_{H}<0$ is often referred to as "catching up to the Joneses", while $U_{H}>0$ can be interpreted as characterizing altruism. ${ }^{9}$

But with agents being forward-looking, particularly with respect to their consumption decisions, there is no reason why the reference level should be based on only past consumption levels. Indeed, it is just as natural for it to be forward-looking (or perhaps combine elements of both). ${ }^{10}$ That is, we assume that utility is of the form

$$
\int_{0}^{\infty} U(C, A) e^{-\beta t} d t
$$

where the reference level, $R$, is now articulated in terms of future consumption anticipations, $A$, so that welfare at each instant depends upon current consumption and anticipated future consumption.

\footnotetext{
8 In their empirical study Houthakker and Taylor draw the distinction between durable consumption and habits, depending on the sign of the consumption stock in the regression of the consumption flow equation. The sign of this coefficient will in turn depend upon the sign of the cross derivative $U_{C H}$ in the underlying utility function. The distinction between habits and durability is emphasized in the context of exchange rate policy by Mansoorian (1998).

${ }^{9}$ One can also interpret the "internal habits" case as representing the preferences of a central planner who internalizes the externalities of the agents, whether they be positive or negative; see Turnovsky and Monteiro (2007).

${ }^{10}$ In principle one could postulate $R$ to be a function of both past and expected future consumptions. For expositional purposes, we prefer to treat them separately.
} 
As for the conventional backward-looking habits case, the forward-looking anticipated consumption reference point, $A$, may be externally or internally generated. In the former case, the individual's well-being depends upon society's expected future consumption profile, whereas in the latter case it depends upon his own personal expected future consumption.

Despite the formal symmetry between backward-looking and forward-looking consumption benchmarks, there are several important differences that need to be considered. First, in the backward-looking case, since $H($.$) as defined in (2c) is based on past observed consumption levels,$ it is clearly known and well-defined for any arbitrary value of $\kappa$. In contrast, the reference point $A$ being based on expectations is unrealized and is inherently uncertain. To preserve comparability with the more familiar backward-looking benchmark we shall specify the anticipation-based reference consumption benchmark at time $t$, by

$$
A(t)=\rho \int_{t}^{\infty} C(s) e^{-\rho(s-t)} d s
$$

the time derivative of which implies

$$
\dot{A}(t)=\rho(A(t)-C(t))
$$

In adopting the specification (3b), with its known weights assumed to decline exponentially into the future, we are clearly abstracting from uncertainty involving anticipated future consumption. A second important difference is that whereas the past cannot be altered, so that its effect on the present is determined by past decisions, anticipations can potentially be influenced by the present. This is a potential source of time inconsistency, from which we shall also abstract by assuming that agents are committed to their initial decisions.

A further issue is that with $A(t)$, as defined in (3b) being forward-looking, to be viable it must be consistent with the agent's (or economy's) intertemporal budget constraint from time $t$ on. It makes no sense for an agent's anticipated future consumption index to be unattainable in the sense 
of being incompatible with the relevant resource constraints. ${ }^{11}$

Letting $w(s)$ and $r(s)$ denote the wage rate and interest rate at time $s, \bar{L}$ denote the labor supply, which we take to be fixed inelastically, and $K(t)$ be the agent's capital stock at time $t$, the intertemporal budget constraint at time $t$ is

$$
\int_{t}^{\infty} C(s) e^{-\int_{t}^{s} r(\tau) d \tau} d s=K(t)+\int_{t}^{\infty} w(s) \bar{L} e^{-\int_{t}^{s} r(\tau) d \tau} d s
$$

Anticipated future consumption must be consistent with this constraint for all $t$. This means that

$$
\frac{A(t)}{\rho}=\int_{t}^{\infty} C(s) e^{-\rho(s-t)} d s \leq \int_{t}^{\infty} C(s) e^{-\int_{t}^{s} r(\tau) d \tau} d s=K(t)+\int_{t}^{\infty} w(s) \bar{L} e^{-\int_{t}^{s} r(\tau) d \tau} d s
$$

and in particular

$$
\frac{A(0)}{\rho}=\int_{0}^{\infty} C(s) e^{-\rho s} d s \leq \int_{0}^{\infty} C(s) e^{-\int_{0}^{s} r(\tau) d \tau} d s=K_{0}+\int_{0}^{\infty} w(s) \bar{L} e^{-\int_{0}^{s} r(\tau) d \tau} d s
$$

This equation emphasizes how the expected lifetime resources of the economy agent constrain its rational anticipations of future consumption. For these consumption expectations to be consistently viable we must have for all $s, t$

$$
e^{-\rho(s-t)} \leq e^{-\int_{t}^{s} r(\tau) d \tau}
$$

which in the limit, as $s \rightarrow \infty$ and $r(s) \rightarrow \beta$ [as will be shown below], reduces to $\rho \geq \beta$, the agent's rate of time preference. If $\beta>\rho$, after some point, the present value of the agent's anticipated consumption exceeds his resources and is unrealizable. ${ }^{12}$ If $\rho=\beta$, the agent's anticipations are constrained by his current wealth, plus future labor income. To the extent that the latter is determined by the time path of current capital, the utility function is of the form $U(C, K)$ and is equivalent to the form of utility function proposed by Kurz (1968) and pursued further in the "spirit of capitalism" literature discussed more recently by Zou (1999) and others. In this regard, we may

11 If $A(t)$ were incompatible with the intertemporal budget constraint, at some point the agent will not realize his expectations and will suffer an unexpected loss of consumption which will need to be taken into account.

${ }^{12}$ Faria and McAdam characterize the condition $\rho>\beta$ as a stability condition. 
note that Kuznitz, Kandel, and Fos (2008) assume $r(t)=r=\rho$ and abstract from labor income. In that case the intertemporal budget constraint reduces to $A(0)=\rho K_{0}$, thereby imposing an initial condition on $A(0)$.

Hence, we shall focus on the case where $\rho>\beta$, in which case the anticipations stock is unconstrained by the agent's intertemporal resources. Constraining $\rho>\beta$ allows us to focus on the interaction of anticipations for the relatively near future with current consumption, arguably the most relevant comparison.

In other respects, the forward-looking internal consumption index, $A$, parallels the backwardlooking index, $H$, in that it embodies two effects. The first is the negative reference effect, $U_{A}<0$, which for the constant elasticity utility function has the property that anticipations are complementary with current consumption, $U_{C A}>0 .{ }^{13}$ The only difference from before is in how the reference consumption index is defined (whether in terms of past, or expected future, consumption levels). The second is the "anticipations effect" which, since A pertains to aggregate consumption that yields positive utility, it is plausible to assume is positive, $U_{A}>0$, with current consumption and anticipations being Edgeworth substitutes, $U_{C A}<0 .{ }^{14}$ As for $U_{C H}$, the overall sign of $U_{C A}$ depends upon which effect dominates.

When the reference consumption index, $A$, is external, $U_{A}<0\left(U_{A}>0\right)$ is the analogue to $U_{H}<0\left(U_{H}>0\right)$ in the conventional backward-looking habit case. In either case if the external reference consumption level increases, while the agent's current consumption level remains unchanged, the effect on utility is qualitatively the same; the only difference is in the construction of the reference consumption level.

13 This assumption was also suggested by Elster and Loewenstein (1992, p.225) who argued that "when the future is expected to be superior to the present, the comparison leads to a denigration of the present." Köszegi and Rabin (2006, 2007 ) adopt a utility function of the form $U(C, A)=V(C)+\mu(C-A), \mu^{\prime}>0, \mu^{\prime \prime} \cdot(C-A)<0$, which implies $U_{A}=-\mu^{\prime}<0, \operatorname{sgn}\left(U_{A C}\right)=\operatorname{sgn}(C-A)$, the latter introducing an asymmetry into the utility gains/losses associated with the deviations in actual consumption from the reference level.

${ }^{14}$ The possibility that the anticipation of a specific unpleasant consumption event (such as a planned visit to the dentist) affecting today's utility adversely, is unlikely to apply to aggregate consumption. We may also note $U_{A}<0$ in the internal reference case may be viewed as characterizing a central planner who internalizes the fact that the representative agent is jealous toward anticipated increases in the external consumption reference level. 


\section{Macroeconomic equilibrium with a forward-looking external consumption reference}

We shall assume that the economy comprises a large number of identical agents, each of whom supplies labor inelastically. The representative agent chooses consumption and the rate of capital accumulation to:

$$
\text { Maximize } \int_{0}^{\infty} U(C, A) e^{-\beta t} d t
$$

subject to

$$
\dot{K}(t)=r(t) K(t)+w(t) \bar{L}-C(t)
$$

Anticipations are generated externally by

$$
\dot{A}(t)=\rho(A(t)-\bar{C}(t))
$$

where $\bar{C}(t)$ denotes the average economy-wide consumption, which together with $A(t)$ are taken as given by the agent. We assume that the agent derives positive, but diminishing, marginal utility from his own consumption, $U_{C}>0, U_{C C}<0$. An increase in the external reference consumption level will raise or lower the agent's welfare depending upon whether he is altruistic or jealous.

The agent's optimality conditions are standard

$$
\begin{aligned}
& U_{C}(C, A)=\lambda \\
& r(t)=\beta-\frac{\dot{\lambda}}{\lambda}
\end{aligned}
$$

where $\lambda(t)$ denotes the agent's marginal utility of wealth. Solving (7a) yields

$$
C=C(\lambda, A)
$$

from which we see that

$$
C_{\lambda}=1 / U_{C C}<0 ; \quad C_{A}=-U_{A C} / U_{C C}
$$


In particular, an increase in the (anticipated) reference consumption level will raise the agent's current consumption if and only if $A$ and $C$ are "Edgeworth complementary".

Firms produce output using a standard neoclassical production function, $F(K, L)$, paying labor and capital their respective marginal products, net of depreciation, $\delta$, in the latter case

$$
w=F_{L}(K, L) \quad r=F_{K}(K, L)-\delta
$$

Thus, combining the household, the production side, and the evolution of anticipated future consumption, and assuming that in equilibrium $C(t)=\bar{C}(t)$, the macrodynamic equilibrium is described by the set of equations

$$
\begin{aligned}
& \dot{K}(t)=F(K, \bar{L})-C(\lambda, A)-\delta K \\
& \dot{\lambda}(t)=\lambda\left[\beta+\delta-F_{K}(K, \bar{L})\right] \\
& \dot{A}(t)=\rho[A(t)-C(t)]
\end{aligned}
$$

In effect, this system is an augmented Ramsey model, in which the standard form is nested in the first two equations. In order to be intertemporally viable

$$
\lim _{t \rightarrow \infty} \lambda(t) K(t) e^{-\beta t}=0
$$

The steady state of this system, obtained when $\dot{K}=\dot{\lambda}=\dot{A}=0$, is described by

$$
\begin{aligned}
& F_{K}(\tilde{K}, \bar{L})=\beta+\delta \\
& \tilde{C}=F(\tilde{K}, \bar{L})-\delta \tilde{K}=\tilde{A}
\end{aligned}
$$

With inelastic labor supply, the steady-state equilibrium quantities of capital, output, and consumption are the same as in the conventional Ramsey model. The capital stock is determined by the modified Golden-rule condition (11a), and given output net of depreciation, consumption is determined by the aggregate market-clearing condition (11b), which in turn determines equilibrium anticipations. The consumption reference level - whether it be external or internal, backward- 
looking or forward-looking - has no long-run effects on equilibrium quantities. However, through (7a) it does affect the long-run marginal utility of wealth, thereby influencing the rate of wealth accumulation and the transitional paths, in potentially profound ways. This property, obtained under time-separable utility by Liu and Turnovsky (2005), extends to this more general set-up, and as in the earlier analysis, is a consequence of the inelastic labor supply. If labor is supplied elastically, these externalities will also have permanent impacts on production and consumption, as we discuss in Section $6 .^{15}$

To analyze the local dynamics along the transitional path, we linearize (9) around (11) to obtain:

$$
\left(\begin{array}{c}
\dot{K}(t) \\
\dot{\lambda}(t) \\
\dot{A}(t)
\end{array}\right)=\left(\begin{array}{ccc}
F_{K}-\delta & -C_{\lambda} & -C_{A} \\
-\tilde{\lambda} F_{K K} & 0 & 0 \\
0 & -\rho C_{\lambda} & \rho\left(1-C_{A}\right)
\end{array}\right)\left(\begin{array}{c}
K(t)-\tilde{K} \\
\lambda(t)-\tilde{\lambda} \\
A(t)-\tilde{A}
\end{array}\right)
$$

In the Appendix we show that this system has one stable eigenvalue, $v_{1}<0$, and two unstable eigenvalues, $v_{2}>0, v_{3}>0$. Imposing the transversality condition, (10), we see that starting from a given initial capital stock, $K_{0}$, the stable local transitional path for capital and its shadow value are

$$
\begin{aligned}
& K(t)-\tilde{K}=\left[K_{0}-\tilde{K}\right] e^{v_{1} t} \\
& \lambda(t)-\tilde{\lambda}=-\frac{\tilde{\lambda} F_{K K}}{v_{1}}[K(t)-\tilde{K}]
\end{aligned}
$$

which are of precisely the same general form as in the conventional Ramsey model. The corresponding adjustment paths for consumption and anticipations are

$$
\begin{aligned}
& C(t)-\tilde{C}=\left(\beta-v_{1}\right)[K(t)-\tilde{K}] \\
& A(t)-\tilde{A}=\frac{\rho}{\left(\rho-v_{1}\right)}[C(t)-\tilde{C}]=\frac{\rho}{\left(\rho-v_{1}\right)}\left(\beta-v_{1}\right)[K(t)-\tilde{K}]
\end{aligned}
$$

\footnotetext{
${ }^{15}$ We should also note that in the presence of technological progress habits affect the steady state even if labor supply is inelastic; see Alvarez-Cuadrado et al (2004) and Wendner (2011).
} 
From (13c) and (13d) we see that

$$
\dot{A}(t)=\frac{\rho}{\left(\rho-v_{1}\right)} \dot{C}(t)=\frac{\rho}{\left(\rho-v_{1}\right)}\left(\beta-v_{1}\right) \dot{K}(t)
$$

Suppose that some structural change, such as an increase in productivity, generates a longrun increase in capital stock, $d \tilde{K}>0$. From (13c) and (13d) we see that on impact

$$
d A(0)-d C(0)=\frac{-\left(\beta-v_{1}\right) v_{1}}{\left(\rho-v_{1}\right)} d \tilde{K}>0
$$

Thus, at any point of time during the transition, current consumption responds more rapidly than do current anticipations to the evolution of the capital stock. This is because following a structural change, the level of anticipations immediately incorporates the expectations of the entire future consumption path, so that $d A(0)>d C(0)$, thus requiring it to adjust less during the subsequent transition. The slower $\rho$, the more forward looking are expectations and the slower its adjustment. We also show in the Appendix that $\left(-v_{1}\right)>\left(-v^{*}\right)>0$, if and only if $U_{C A}>0$, where $v^{*}$ denotes the stable eigenvalue in the basic Ramsey model. This enables us to state the following:

Proposition 1: The introduction of an external forward-looking consumption reference will decrease (increase) the rate of convergence (described by $-v_{1}$ according to whether expectations of future consumption decrease (increase) the marginal utility of current consumption. This in turn will imply that capital will be accumulated less (more) rapidly to make up the current shortfall in the capital stock and that current consumption will react slower (faster) to the accumulation of capital.

The following intuition applies. If $U_{C A}<0$, the forward-looking external consumption reference reduces the marginal utility of wealth at each instant of time, thereby reducing the incentive to accumulate capital, thus slowing down the transitional adjustment.

In our numerical simulations we shall compare the equilibrium dynamics corresponding to an external forward-looking consumption reference to the transition in the conventional case where the 
reference is backward-looking. As is well known from previous work, the stable manifold is twodimensional with the two sluggish variables, capital and habits serving as state variables. ${ }^{16}$ Proposition 1 has one interesting implication for this comparison. One of the criticisms of the basic Ramsey model is that it generates an implausibly rapid speed of convergence, often around $10 \%$ or more at annual rates. Alvarez-Cuadrado et al. (2004) find that introducing backward-looking external habits having the property $U_{H C}<0$, consistent with habit formation, has the undesirable effect of increasing the rate of convergence, unless the habits evolve unrealistically slowly. By contrast, introducing the corresponding forward-looking external habits $\left(U_{A C}<0\right)$ reduces the speed of convergence, thereby bringing it in closer agreement to the empirical evidence.

\section{Macroeconomic equilibrium with forward-looking internal consumption reference}

We now turn to the case where the forward-looking consumption reference is inwardlooking, being based on the agent's anticipations of his own future consumption levels. In this case the agent's optimization problem is to maximize (6a), subject to (6b) and $\left(6 c^{\prime}\right)^{17}$

$$
\dot{A}(t)=\rho(A(t)-C(t))
$$

so that the agent internalizes the evolution of anticipations. The optimality conditions now become

$$
\begin{aligned}
& U_{C}(C, A)=\lambda+\rho \mu \\
& r(t)=\beta-\frac{\dot{\lambda}}{\lambda} \\
& \frac{U_{A}(C, A)}{\mu}+\rho=\beta-\frac{\dot{\mu}}{\mu}
\end{aligned}
$$

where $\mu$ denotes the shadow value of anticipations. As usual, the following transversality conditions at infinity must hold

$$
\lim _{t \rightarrow \infty} \lambda K e^{-\beta t}=0
$$

\footnotetext{
${ }^{16}$ Since this case is familiar, we simply report the equilibrium dynamics in the Appendix.

${ }^{17}$ For notational convenience we omit the inelastically fixed labor supply, $\tilde{L}$ from the functions.
} 


$$
\lim _{t \rightarrow \infty} \mu A e^{-\beta t}=0
$$

But, in addition, since we are assuming that $\rho>\beta$ so that anticipations are weighted relatively heavily to the near future, and therefore are not bound by the agent's lifetime resources, the initial anticipations $A(0)$ can therefore be chosen as part of the overall optimization, though subject to the constraint $\left(6 c^{\prime}\right)$. This implies that their associated initial shadow value is $\mu(0)=0$; see e.g. Hestenes (1966), Leonard and Long (1992), Kamien and Schwartz (1971). That is, agents set their initial expectations so that their shadow value is zero.

The optimality conditions (15) have the following interpretation. First, (15a) equates the marginal utility of current consumption to its cost, which now comprises two components. The first is the usual shadow value of the current capital foregone. But in addition, the shadow value of future anticipated consumption, $\rho \mu$, must also be taken into account. An increase in the latter shadow value of anticipations induces the agent to reduce current consumption thereby enabling him to prolong his enjoyment of anticipations. This term incorporates the delay of current consumption in response to anticipations, emphasized by Loewenstein (1987). Equation (15b) corresponds to (7b), while (15c) now is an arbitrage condition linking the rate of return on anticipations, given by the left hand side to that of current consumption, expressed in terms of units of anticipations.

Since this last equation is crucial, but less familiar, it is worth interpreting it using first principles. Imagine an individual choosing to "invest" a unit of resources in anticipations at some arbitrary time $t$. The cost of doing so equals the shadow value of anticipations at time $t$, namely $\mu(t)$. The benefits achieved at time $t+d t$, comprise the utility benefits derived over the period $(t, t+d t), U_{A}(t) d t$, together with the value of the anticipations at time $t+d t$. These are given by $(1+\rho d t) e^{-\beta d t} \mu(t+d t)$, which measures the enhanced quantity, discounted for the fact it is at $d t$ time units later, and revalued at the price at that time. Equating the costs and benefits yields

$$
U_{A}(t) d t+(1+\rho d t) e^{-\beta d t} \mu(t+d t)=\mu(t)
$$

Now using the fact that $e^{-\beta d t}=1-\beta d t+o(d t), \mu(t+d t)=\mu(t)+\dot{\mu}(t) d t+o(d t)$, where $o(d t)$ denotes terms of order smaller than $d t$, we may expand (17) in the form 


$$
U_{A}(t) d t+[\mu(t)+[\mu(t)(\rho-\beta)+\dot{\mu}(t)] d t+o(d t)]=\mu(t)
$$

Subtracting $\mu(t)$ from both sides, dividing by $d t$, and using the property that $\lim _{d t \rightarrow 0}(o(d t) / d t)=0$, yields (15c).

From equation (15a) we now obtain

$$
C=C(A, \lambda, \mu)
$$

where

$$
C_{A}=-\frac{U_{C A}}{U_{C C}}, \quad C_{\lambda}=\frac{1}{U_{C C}}<0, \quad C_{\mu}=\frac{\rho}{U_{C C}}<0
$$

The macroeconomic equilibrium is summarized by

$$
\begin{aligned}
& \dot{K}(t)=F(K)-C(\lambda, A, \mu)-\delta K \\
& \dot{\lambda}=\lambda\left[\beta+\delta-F_{K}(K)\right] \\
& \dot{A}=\rho(A-C(A, \lambda, \mu)) \\
& \dot{\mu}=(\beta-\rho) \mu-U_{A}(C(A, \lambda, \mu), A)
\end{aligned}
$$

The problem is somewhat unconventional, in that anticipations, although postulated to evolve in accordance with a differential equation, (6c), are not sluggish, in that their initial value, $A(0)$, can be freely chosen, with the consequence that it is its shadow value that becomes predetermined at $\mu(0)=0$. Nevertheless, we still need to ensure that the transversality condition, (16b), is met. To do this, we first solve (18c) and (18d), expressing the solutions in the form

$$
\begin{aligned}
& A(t)=e^{\rho t}\left[A(0)-\rho \int_{0}^{t} C(s) e^{-\rho s} d s\right] \\
& \mu(t)=e^{(\beta-\rho) t}\left[\mu(0)-\int_{0}^{t} U_{A}(s) e^{-(\beta-\rho) s} d s\right]
\end{aligned}
$$

Applying the transversality condition and using the fact that $\mu(0)=0$, we find 


$$
\lim _{t \rightarrow \infty}\left\{\left[A(0)-\rho \int_{0}^{t} C(s) e^{-\rho s} d s\right]\left[\int_{0}^{t} U_{A}(s) e^{-(\beta-\rho) s} d s\right]\right\}=0
$$

Since we are assuming $\rho>\beta$, this condition will be met if and only

$$
A(0)=\rho \int_{0}^{\infty} C(s) e^{-\rho s} d s
$$

consistent with the first equality in (5).

Steady state, obtained when $\dot{K}=\dot{A}=\dot{\lambda}=\dot{\mu}=0$, is described by

$$
\begin{aligned}
& F(\tilde{K})=\tilde{C}+\delta \tilde{K} \\
& F_{K}(\tilde{K})=\beta+\delta \\
& \tilde{A}=\tilde{C} \\
& U_{C}(\tilde{C}, \tilde{A})=\tilde{\lambda}+\rho \tilde{\mu} \\
& (\beta-\rho) \tilde{\mu}-U_{A}(\tilde{C}, \tilde{A})=0
\end{aligned}
$$

and determine $(\tilde{K}, \tilde{A}, \tilde{C}, \tilde{\lambda}, \tilde{\mu})$. The first three equations correspond to (11a) and (11b) for the external reference point case, while (19d) restates (15a). The final equation implies that with $\rho>\beta$, if expected future consumption yields positive marginal utility its steady-state shadow value must be negative and vice versa. This result is a reflection of a key assumption that we are making, namely that agents fully commit to their initial optimality conditions set at time $t=0$. With agents being free to choose initial expectations, this raises the potential issue of time inconsistency, in that if they were free to renege they would like to choose $A$ such that $\mu(t)=0$ at each instant of time. This expression for $\mu$ represents the accumulated losses due to the fact that we are setting $\mu(0)=0$ only at time zero and not re-optimizing thereafter, as agents would really like to do. With $\rho>\beta$, starting from time zero they are accumulating anticipations at too fast a rate so that the only way the equilibrium arbitrage condition can hold is if the positive (negative) marginal utility of anticipations is assigned a negative (positive) value. 
If we combine (19d) and (19e) from the steady-state conditions we get:

$$
\tilde{\lambda}=U_{C}+\frac{\rho}{\rho-\beta} U_{A}
$$

This equation asserts that in contrast to the conventional model (where $\tilde{\lambda}=U_{C}$ ) in equilibrium the shadow value of capital must equal the current marginal utility of consumption (the first term) plus the discounted marginal utility derived from anticipations (the second term).

Finally, we observe that the steady-state levels of capital, output, and consumption are unaffected by the presence of the consumption reference point, which will, however, affect the transitional dynamics. Moreover, $A$ will affect both the transition and steady state if labor supply is elastic; see Section 6 where we discuss the steady state.

\subsection{Transitional Dynamics}

We now turn to the transitional dynamics and to do so we linearize (18) around the steady state:

$$
\left(\begin{array}{c}
\dot{K} \\
\dot{\mu} \\
\dot{\lambda} \\
\dot{A}
\end{array}\right)=\left(\begin{array}{cccc}
F_{K}-\delta & -C_{\mu} & -C_{\lambda} & -C_{A} \\
0 & (\beta-\rho)-U_{A C} C_{\mu} & -U_{A C} C_{\lambda} & -\left[U_{A A}+U_{A C} C_{A}\right] \\
-F_{K K} & 0 & 0 & 0 \\
0 & -\rho C_{\mu} & -\rho C_{\lambda} & \rho\left(1-C_{A}\right)
\end{array}\right)\left(\begin{array}{c}
K-\tilde{K} \\
\mu-\tilde{\mu} \\
\lambda-\tilde{\lambda} \\
A-\tilde{A}
\end{array}\right)
$$

These depend upon the characteristic equation to (21), which we write in the compact form

$$
D(v) \equiv v^{4}-2 \beta v^{3}+\theta_{2} v^{2}+\theta_{3} v+\frac{F_{K K}}{U_{C C}} \rho(\rho-\beta)=0
$$

where the solutions, $v_{i}, i=1, \ldots, 4$, are the eigenvalues. For notational convenience we let

$$
\begin{aligned}
& \theta_{2} \equiv \beta^{2}+\left[\rho\left(1-C_{A}\right)\left[\beta-\rho\left(1-C_{A}\right)\right]-\rho C_{\mu} U\right]-\frac{F_{K K}}{U_{C C}} \equiv \Omega+\beta^{2} \\
& \theta_{3} \equiv-\beta\left[\rho\left(1-C_{A}\right)\left[\beta-\rho\left(1-C_{A}\right)\right]-\rho C_{\mu} U\right]+\beta \frac{F_{K K}}{U_{C C}} \equiv-\beta \Omega
\end{aligned}
$$


With both $C, A$ being subject to the agent's choice, to proceed it is convenient to impose further assumptions on $U$ pertaining to $A$. The first is that $U$ is concave in both $C, A$, implying ${ }^{18}$

$$
U \equiv U_{A A}+U_{A C} C_{A}=\frac{U_{A A} U_{C C}-U_{A C}^{2}}{U_{C C}}<0
$$

It is immediately apparent from (22) that the sum of the eigenvalues, $v_{1}+v_{2}+v_{3}+v_{4}=2 \beta>0$, while the product, $v_{1} v_{2} v_{3} v_{4}=\left(F_{K K} / U_{C C}\right) \rho(\rho-\beta)>0$, which implies that either all eigenvalues are positive, or that there are two positive and two negative. Which of these two cases prevails can be determined by applying Descartes rule of signs to (22). ${ }^{19}$

To have four positive eigenvalues, Descartes rule of sign would require four sign changes in the coefficients of the polynomial (22), and hence $\theta_{2}>0, \theta_{3}<0$, or equivalently $\Omega+\beta^{2}>0, \Omega>0$. But adding weak conditions, we can show $\Omega<0$, thereby ruling out this sign pattern. To see this, we may rewrite

$$
\Omega \equiv \rho\left(1-C_{A}\right)\left[\beta-\rho\left(1-C_{A}\right)\right]-\rho C_{\mu} U-\frac{F_{K K}}{U_{C C}}=\frac{\rho}{U_{C C}}(\beta-\rho)\left(U_{C C}+U_{C A}\right)-\frac{\rho^{2}}{U_{C C}}\left(U_{C A}+U_{A A}\right)-\frac{F_{K K}}{U_{C C}}
$$

Weak (sufficient) conditions to ensure $\Omega<0$ are

$$
U_{C C}+U_{C A} \leq 0, U_{A A}+U_{C A} \leq 0 \quad \text { (diminishing marginal utilities dominate cross effects) }
$$

in which case (22) involves only two sign changes and therefore implies two positive and two negative eigenvalues. ${ }^{20}$

We still must check that the transversality conditions, (16), are met. This will be so if and only $2 v_{i}<\beta$, which clearly holds for the two negative roots, while by examining (22) we can show that it is violated for the two positive roots. ${ }^{21}$ With two stable eigenvalues, the intertemporally

\footnotetext{
${ }^{18}$ We should note that Alonso-Carrera et al (2005) have identified problems associated with concavity in models of habit formation. The restrictions we are proposing are sufficient for a well-behaved saddlepoint solution. They are not necessary and indeed a well defined equilibrium dynamic path can be obtained under weaker conditions.

${ }^{19}$ Descartes rule of signs states that number of positive roots to the equation $x^{n}+a_{1} x^{n-1}+a_{2} x^{n-2}+\ldots+a_{n-1} x+a_{n}=0$ equals the number of sign changes between the successive coefficients.

${ }^{20}$ These conditions are met by our simulations. They are also met by the assumption of diminishing sensitivity imposed by Köszegi and Rabin $(2006,2007)$.

${ }^{21}$ With regard to the two positive roots, we can determine whether this is met by considering $D(\beta), D^{\prime}(\beta)$. Evaluating
} 
viable formal solution for the local dynamics is of the form

$$
\begin{aligned}
& K(t)-\tilde{K}=B_{1} e^{v_{1} t}+B_{2} e^{v_{2} t} \\
& \mu(t)-\tilde{\mu}=B_{1} \omega_{21} e^{v_{1} t}+B_{2} \omega_{22} e^{v_{2} t} \\
& \lambda(t)-\tilde{\lambda}=B_{1} \omega_{31} e^{v_{1} t}+B_{2} \omega_{32} e^{v_{2} t} \\
& A(t)-\tilde{A}=B_{1} \omega_{41} e^{v_{1} t}+B_{2} \omega_{42} e^{v_{2} t}
\end{aligned}
$$

where $B_{1}, B_{2}$ are arbitrary, $K(0)=K_{0}, \mu(0)=0$, and the vector $\left(1, \omega_{2 i}, \omega_{3 i}, \omega_{4 i}\right), i=1,2$ is the normalized eigenvector associated with the stable eigenvalue $v_{i}$. That is $\left(1, \omega_{2 i}, \omega_{3 i}, \omega_{4 i}\right)$ satisfies

$$
\left(\begin{array}{cccc}
\beta-v_{i} & -C_{\mu} & -C_{\lambda} & -C_{A} \\
0 & (\beta-\mu)-U_{A C} C_{\mu}-v_{i} & -U_{A C} C_{\lambda} & -\left[U_{A A}+U_{A C} C_{A}\right] \\
-F_{K K} & 0 & -v_{i} & 0 \\
0 & -\rho C_{\mu} & -\rho C_{\lambda} & \rho\left(1-C_{A}\right)-v_{i}
\end{array}\right)\left(\begin{array}{c}
1 \\
\omega_{2 i} \\
\omega_{3 i} \\
\omega_{4 i}
\end{array}\right)=0
$$

The constants $B_{1}, B_{2}$ are obtained by imposing the initial conditions $K(0)=K_{0}, \mu(0)=0$

$$
\begin{aligned}
& B+B_{2}=K_{0}-\tilde{K} \\
& \omega_{21} B_{1}+\omega_{22} B_{2}=-\tilde{\mu}
\end{aligned}
$$

and will depend upon the shock.

Finally, the traditional backward-looking solution corresponding to habits is reported in the Appendix. There it is seen that the characteristic equation is of exactly the same form as (22), except now $\kappa=-\rho>0$. This is shown to have two stable and two unstable roots, yielding a saddlepoint, with $K, H$ being conventional state variables. With labor supply being inelastic, the steady-state solutions for capital, output, and consumption are as in (19) enabling us to state:

these expressions, we obtain $D(\beta)=\left(F_{K K} / U_{C C}\right) \rho(\rho-\beta)>0, D^{\prime}(\beta)=\beta \Omega<0$, which implies that both positive roots $v_{i}>\beta$, violating the transversality conditions. 
Proposition 2: With inelastic labor supply, steady-state consumption and output is identical to that of conventional Ramsey model. It is independent of any consumption reference point, either based on past habits or future anticipations.

\section{Numerical Simulations}

\subsection{Calibration}

To obtain further insights into the role of anticipated future consumption and the contrasting role of the two specifications of the reference consumption levels on the transitional dynamics we must proceed numerically. To do so, we adopt specific production and utility functions, and for this purpose we assume a Cobb-Douglas function for production and a constant elasticity utility function for preferences

$$
\begin{aligned}
& Y=\alpha K^{1-\sigma} \bar{L}^{\sigma} \\
& U=\frac{1}{1-\varepsilon}\left(C R^{\theta}\right)^{1-\varepsilon}
\end{aligned}
$$

where in (25b) $\theta$ parameterizes the impact of the reference consumption level, while $1 / \varepsilon$ is the intertemporal elasticity of substitution. $^{22}$

To derive the transitional dynamics we need to further specify parameter values we employ in the simulations. These are summarized in Table 1.A and are mostly standard and noncontroversial. In this respect $\sigma=0.65$, implying a labor share of income of $65 \%$, rate of time preference $\beta=0.04$, intertemporal elasticity of substitution $1 / \varepsilon=0.4$, and depreciation rate of $\delta=0.075$, are well documented, while being a neoclassical model, the normalization of the productivity level $\alpha=1$ is unimportant.

The critical parameters relate to $\theta$, the impact of the reference stock, $R$, and $\rho(\kappa)$ the speed with which it adjusted. We consider two benchmarks, $\theta=0.5$ ( $R$ impacts utility positively),

\footnotetext{
${ }^{22}$ In our numerical work we have generalized both functions to the CES form, but it turns out that our numerical results are robust with respect to plausible ranges of the (intratemporal) elasticity of substitution and hence we restrict ourselves to these simpler forms.
} 
and $\theta=-0.5$ ( $R$ impacts utility negatively). For $\varepsilon=2.5>1$ these parameters also imply that $U_{R C}<0, U_{R C}>0$, respectively. To preserve comparability between the forward-looking and backward-looking reference stock, we maintain the same value for the two cases $R=A, H$. In terms of the two effects that we identified earlier, $\theta=0.5$ implies that the anticipations effect dominates in the case $R=A$, while the durability effect dominates if $R=H$. In contrast, if $\theta=-0.5$ the reference effect dominates in both cases. Also to preserve comparability we assume the same speed of adjustment for these two cases and thus set $\rho=0.2, \kappa=0.2$. These benchmark values correspond to those of Carroll et al (1997) and Alvarez-Cuadrado et al (2004).

The first line in Table 2 summarizes the base equilibrium values of consumption and capital corresponding to the benchmark parameters. These can be shown to imply an initial output-capital ratio of 0.30 and a consumption-output ratio of 0.783 . Starting from this initial equilibrium, we compare the dynamic adjustment following a $20 \%$ increase in productivity as represented by an increase in $\alpha$ from 1 to 1.2 . It can be immediately shown that with labor supply fixed, in all cases it leads to long-run proportional increases in capital and consumption of around $32 \%$. However, the transitional paths whereby these common long-run adjustments are reached contrast sharply, particularly in the case where the reference point is generated internally.

\subsection{Transitional dynamics with externally generated reference point}

As a benchmark, we begin with the conventional Ramsey model, illustrated by the solid line. In that case the immediate effect of an increase in productivity $\alpha$ is to raise the return to capital, thereby inducing an increase in capital accumulation, so that $\dot{K}(0)$ increases to 0.129 , implying an initial growth rate of capital of $2.02 \%$. At the same time, it increases the time path of expected future output, which in effect raises wealth, inducing an immediate increase in consumption from its initial steady-state level of 1.498 to 1.751 , or a $16.9 \%$ increase. Thereafter, the ongoing accumulation of the capital stock causes consumption to continue to increase and both capital and consumption increase monotonically to their respective new higher steady-state levels of 8.438 and 1.983, as illustrated in both Figs. 1 and 2. 
Now, suppose that the agent's utility is positively affected by the anticipation of economywide future consumption $(\theta=0.5)$. The dynamics are illustrated by the short-dashed lines in the left hand panel. With consumption increasing monotonically over time, the initial response of future consumption anticipations, $A(0)$, being the discounted sum of all expected future consumption levels, is to increase by more than does $C(0)$, namely to $1.829(22.1 \%)$, as compared to 1.787 , for the latter. At the same time $\theta=0.5>0$ implies $U_{C A}<0$, and the anticipations reduce the marginal utility of consumption and therefore the marginal utility of wealth. This reduces the incentive to accumulate capital and as a result the initial increase in the rate of capital accumulation, $\dot{K}(0)$, is reduced from 0.129 to 0.113 , or $1.77 \%$. In order for product market equilibrium to be maintained this requires an offsetting increase in the initial rate of consumption and $C(0)$ increases from 1.751 (in the Ramsey economy) to 1.787. During the subsequent transition, consumption, anticipations, and capital all converge at the same common rate, though slower than in the Ramsey economy.

Suppose now agents' utility is positively affected by economy-wide past consumption habits. The dynamics are illustrated by the long-dashed line in the left hand panel. The main difference is that with habits being backward-looking, they evolve gradually from their initial steady-state level, as illustrated in the lowest panel. As a result, being predetermined at time 0 , even though $\theta=0.5>0$ implies $U_{C H}<0$, habits do not have the adverse initial impact on consumption, that anticipations have and therefore on impact consumption increases even more to 1.824 , while the rate of capital accumulation is reduced further to 0.056 , implying a growth rate of $0.88 \%$. However, as habits start to increase following the increase in initial consumption, the marginal utility of consumption declines and there is a slight decline in current consumption, which is offset by an increase in the rate of capital accumulation.

The right hand panels of Fig. 1 illustrates the case $\theta=-0.5$, so that the external consumption reference has an adverse effect on utility, but a positive impact on the marginal utility of current consumption. In this case, which corresponds to the conventional assumption in the "habits literature", the relative short-run responses of consumption and capital accumulation under the three alternative scenarios are reversed 
Further insight into the different initial responses can be obtained by focusing on the initial effect of the productivity increase on the short-run market clearing conditions in the three cases:

$$
\begin{aligned}
& d \dot{K}^{R A}(0)=F\left(K_{0}\right) d \alpha-C_{\lambda}^{R A} d \lambda^{R A}(0) \\
& d \dot{K}^{A}(0)=F\left(K_{0}\right) d \alpha-C_{\lambda}^{A} d \lambda^{A}(0)-C_{A} d A(0) \\
& d \dot{K}^{H}(0)=F\left(K_{0}\right) d \alpha-C_{\lambda}^{H} d \lambda^{H}(0)
\end{aligned}
$$

where the superscripts " $R A$ ", “ $A$ ", " $H$ " denote "Ramsey”, "anticipations", and "habits" respectively. The positive productivity effects are common in all cases, while the initial changes in the marginal utility of wealth, $d \lambda(0)$ are approximately equal (although the levels in the Ramsey model are different). The differences are due to (i) differences in the effect of the marginal utility of wealth on consumption in the Ramsey model from the corresponding effects in the two reference models (which are approximately equal), and (ii) the fact that anticipations being free to respond instantaneously have an immediate effect on consumption and therefore on capital accumulation.

\subsection{Transitional dynamics with internally generated reference point}

Fig. 2 illustrates the case where the consumption reference point is internally generated. We again include the conventional Ramsey model as a benchmark. As noted, the difference between the internally generated and externally generated reference point is that the agent now takes full account of the shadow value of the reference consumption level, given by (18d) or (A.9d), depending upon whether it is forward-looking or backward-looking. Comparing the dynamic paths corresponding to habits in Figs 1 and 2, they can be seen to track one another pretty closely. This confirms the findings of Carroll et al (1997) and Alvarez-Cuadrado et al (2004) who also found this to be the case.

On the other hand, the dynamic paths followed when the internal consumption reference point is based on anticipations contrasts sharply with those obtained when the reference point is 
derived externally. The reason for this is the initial jump in the shadow value, which the inwardlooking agent takes fully into account.

Consider first the case $\theta=0.5$ illustrated in the left hand panel of Fig. 2, we see that the initial steady state implies an initial equilibrium shadow value of anticipations of $\tilde{\mu}=-0.84$. This corresponds to the most plausible case where the agent derives positive current utility from his own anticipated future consumption. With $\varepsilon>1$ this also implies that $U_{C A}<0$, so that anticipations of future his future consumption reduce the utility of current consumption; anticipated future and current consumption are therefore Edgeworth substitutes.

As discussed in Section 4.2, the immediate response following the increase in productivity is for $\mu^{A}(0)=0$, or in other words $d \mu^{A}(0)=0.84$. The consequences for the initial rate of capital accumulation can be seen by taking the differential of the product market equilibrium relationship

$$
d \dot{K}^{A}(0)=F\left(K_{0}\right) d \alpha-C_{\lambda}^{A} d \lambda^{A}(0)-C_{A} d A(0)-\rho C_{\lambda}^{A} d \mu^{A}(0)
$$

With $C_{\mu}^{A}=\rho C_{\lambda}^{A}=\rho\left(U_{C C}\right)^{-1}<0,\left(26 b^{\prime}\right)$ implies that the sharp increase in $\mu(0)$ reduces consumption and increases the rate of capital accumulation. Indeed the sharp increase $d \mu^{A}(0)=0.84$ dominates all other effects, and on impact consumption declines by 5.6\% from 1.498 to 1.414 and correspondingly the initial increase in $\dot{K}(0)$ to 0.466 is equivalent to a growth rate of $7.22 \%$, which exceeds the initial growth rate in the Ramsey economy. Compared to the latter, the fact anticipations yield positive utility provides an incentive for agents to reduce their current consumption in favor of future consumption and prolong the period over which they can enjoy the anticipations. Over time, as capital is accumulated, the agent's current consumption increases, the utility of anticipations declines, as does its shadow value, and eventually $\mu^{A}$ converges to -0.34 .

The right hand panels of Fig 2 assume $\theta=-0.5$, in which case $U_{A}<0, U_{A C}>0$. In this case the negative reference effect dominates the positive anticipations effect and overall agents get disutility from the process of anticipating their own future consumption. This is quite distinct from any discounting for the future. Because anticipations yield negative utility, the agent seeks to reduce these by increasing current consumption dramatically. This not only reduces the resources available 
for future consumption, which can therefore be anticipated, but it also reduces the amount of resources available for investment, which therefore declines. Over time, as the effects of the higher productivity are accumulated, and the adverse effects of anticipations decline, the reductions in capital and consumption are arrested and they begin to increase toward their new higher equilibrium levels. In this case the non-monotonic time path characterizing the transitional dynamics of the anticipations model suggests a sharp contrast with the monotonic adjustment followed by the habits model.

\subsection{Expectations vs. habits}

As a matter of practice, the standard habits model treats consumption as non-durable so that the negative reference effect dominates. Likewise, the pure anticipations model does not consider future anticipations as providing any kind of benchmark, in which case the anticipations effect prevails. Thus it also of interest to contrast the two models by comparing $\theta=-0.5$ in the former case with $\theta=0.5$ in the latter, thereby normalizing their respective intensities in the two cases. This can be seen by comparing the short dashed line in the left hand panel with the long dashed line in the right hand panel of Fig. 2. In this case, both approaches imply monotonic adjustments, although with substantially different speeds of adjustment. Consistent with the previous result obtained by Alvarez-Cuadrado et al. (2004), for these benchmark parameters the habits model implies an overly rapid speed of convergence of almost $10 \%$ per annum, faster than the basic Ramsey model of $6.3 \%$. In contrast, the anticipations model reduces the asymptotic speed of convergence to just $5 \%$, which is more in line with the empirical evidence. In this respect, introducing consumption reference as a forward-looking variable performs more satisfactorily than does the habits model.

\subsection{Sensitivity Analysis}

We should point out that the purpose of our numerical simulations is primarily to illustrate plausible transitional, rather than to replicate a specific economy at some given point in time. In this regard, while the parameters relating to the basic Ramsey model are well established, information on 
the parameters pertaining to the reference consumption stock is sparse. For this reason we have undertaken extensive sensitivity analysis with respect to $\theta, \rho, \kappa$.

The overall conclusion is that our results are generally robust with respect to plausible parameter changes, and because the "Backward-looking" case has been extensively analyzed in AlvarezCuadrado et al (2004), here we report only the main results regarding the impact on the eigenvalues and the transitional dynamics for the "Forward-looking" case ${ }^{23}$. In addition, we present only the results for the case $\theta<0$, with all the results being reversed if $\theta>0$.

Starting with the eigenvalues, we can say that in general, the relation between $\rho$ and the speed of convergence of the real variables is nonmonotonic, with the asymptotic speed of convergence of the overall system increasing with $\rho$ for low values of $\rho$, and decreasing with $\rho$ for high values of $\rho$. Our simulations suggest that if $\theta<0$ which corresponds to the conventional assumption in the "habits literature", the economy with external consumption reference converges slower than the one with internal consumption reference ${ }^{24}$.

Looking at the transitional dynamics, our simulation show that the effect of increasing either the speed of adjustment in the reference stock, $\rho$, or the weight of the reference stock on utility, $\theta$, has the same effect on the immediate response of key variables. Comparing the impact of those parameters on the inward-looking and the outward-looking case, our simulations show the effects are exactly the opposite; thus contrasting with what happens in the "Backward-Looking" case, where the effect is the same for both cases. Intuitively the difference in the "Forward-Looking" case is because the initial jump in the shadow value of anticipation is fully taken into account by the inward-looking agent.

When the agent is inward-looking an increase in either $\rho$ or $\theta$, increases both the initial rate of consumption $C(0)$ and $A(0)$. The initial increase in the response of $C(0)$, reduces the incentive to accumulate capital, thus reducing (i.e. making it more negative) the initial response in the rate of capital accumulation. The main difference between the effect of both parameters is the transitional

\footnotetext{
${ }^{23}$ Further detail is available on request from the authors.

${ }^{24}$ It is worth noticing that these results are contrary to what happens in the "Backward-Looking" case, where the economy with outward looking agents converges faster than the one having inward-looking agents. See section 5.4 for an explanation why this happens.
} 
dynamics, because an increase in the weight of the reference stock on utility, $\theta$, prolongs the duration of the shock, i.e. longer transition, whereas an increase in the speed of adjustment in the reference stock, $\rho$ reduces the duration of the transition because of the faster adjustment of consumption. When the agent is outward-looking the initial jump in the shadow value of anticipation is not internalized, and thus the effects are reversed.

\section{Elastic labor supply}

Thus far we have followed much of the growth literature, and more specifically the "habit formation" literature, and assumed that labor is supplied inelastically. A consequence of this is the steady-state levels of real activity (capital, output, and consumption) are all independent of the reference consumption level, whether it be determined from past consumption, anticipated future consumption, externally, or internally. In all cases, its only impact is on the transition, a finding that is consistent with Liu and Turnovsky's (2005) analysis of "keeping up with the Joneses".

In this section we briefly discuss the long-run consequences of endogenizing labor supply. The critical differences are (i) that the instantaneous utility function is now modified to $U(C, R, L)$, where $R=A, H$, and (ii) the optimality condition with respect to labor supply satisfies the standard optimality condition $U_{L}(C, R, L)=-\lambda F_{L}(K, L)$. Whether the consumption reference is formed from the past or the anticipated future, in steady state, $\tilde{R}=\tilde{A}=\tilde{H}=\tilde{C}$ and the steady state always includes the following three relationships:

$$
\begin{aligned}
& F(\tilde{K}, \tilde{L})=\tilde{C}+\delta \tilde{K} \\
& \beta+\delta=F_{K}(\tilde{K}, \tilde{L}) \\
& U_{L}(\tilde{C}, \tilde{C}, \tilde{L})=-\tilde{\lambda} F_{L}(\tilde{K}, \tilde{L})
\end{aligned}
$$

Equations (25a) and (25b), together with the homogeneity of the production function, imply that the $\operatorname{ratios} \tilde{K} / \tilde{L}, \tilde{Y} / \tilde{L}, \tilde{C} / \tilde{L}$ are independent of any characteristics of the consumption-reference level.

In the case that $R$ is determined externally, the following additional condition must hold: 


$$
U_{C}(\tilde{C}, \tilde{C}, \tilde{L})=\tilde{\lambda}
$$

Combining (25c) and (25d) in that case yields

$$
F_{L}(\tilde{K}, \tilde{L})=-\frac{U_{L}(\tilde{C}, \tilde{C}, \tilde{L})}{U_{C}(\tilde{C}, \tilde{C}, \tilde{L})}
$$

and together (25a), (25b) and (26) determine the steady-state values of $\tilde{K}, \tilde{L}, \tilde{C}$. With the left hand side of (26) being independent of $R$, we immediately see that a necessary and sufficient condition for an external reference to have no effect on real activity is that it has no effect on the marginal rate of substitution between consumption and leisure.

In the case where $R$ is determined internally, in addition to (25a) - (25c) one the following two pairs of conditions must hold, depending upon whether $R$ is forward- or backward-looking:

(i) Forward-looking

$$
\begin{aligned}
& U_{C}(\tilde{C}, \tilde{C}, \tilde{L})=\tilde{\lambda}+\rho \tilde{\mu} \\
& (\beta-\rho) \tilde{\mu}-U_{A}(\tilde{C}, \tilde{C}, \tilde{L})=0
\end{aligned}
$$

(i) Backward-looking

$$
\begin{aligned}
& U_{C}(\tilde{C}, \tilde{C}, \tilde{L})=\tilde{\lambda}-\rho \tilde{\kappa} \\
& (\beta+\rho) \tilde{\kappa}-U_{H}(\tilde{C}, \tilde{C}, \tilde{L})=0
\end{aligned}
$$

Combining these two equations with (25c) yields the following

$$
\begin{gathered}
\text { Forward-looking } \quad F_{L}(\tilde{K}, \tilde{L})=-\frac{U_{L}(\tilde{C}, \tilde{C}, \tilde{L})}{\left[U_{C}(\tilde{C}, \tilde{C}, \tilde{L})+\frac{\rho}{\rho-\beta} U_{A}(\tilde{C}, \tilde{C}, \tilde{L})\right]} \\
\text { Backward-looking } \quad F_{L}(\tilde{K}, \tilde{L})=-\frac{U_{L}(\tilde{C}, \tilde{C}, \tilde{L})}{\left[U_{C}(\tilde{C}, \tilde{C}, \tilde{L})+\frac{\kappa}{\kappa+\beta} U_{H}(\tilde{C}, \tilde{C}, \tilde{L})\right]}
\end{gathered}
$$


From (26') and (26") it is clear that, except in polar cases, an internally generated consumption reference point will indeed affect long-run real activity when labor is supplied elastically.

To obtain further insight, we shall consider the widely adopted constant elasticity utility function

$$
U=\frac{1}{\gamma} C^{\gamma} R^{\theta \gamma}(1-L)^{\alpha \gamma} ; \quad R=A, H
$$

which when applied to (26) immediately implies that the external reference has no long-run effect on real activity.

Applying (27) to (26') and (26") we can express these relationships in the form

$$
\begin{aligned}
& \frac{\chi}{F_{L}} \frac{\tilde{C}}{\tilde{L}}=\frac{(1-\tilde{L})}{\tilde{L}}\left[1+\frac{\rho \theta}{\rho-\beta}\right] \\
& \frac{\chi}{F_{L}} \frac{\tilde{C}}{\tilde{L}}=\frac{(1-\tilde{L})}{\tilde{L}}\left[1+\frac{\kappa \theta}{\kappa+\beta}\right]
\end{aligned}
$$

Given the constancy of $\tilde{C} / \tilde{L}$, we obtain

$$
\begin{aligned}
& \frac{1}{(1-\tilde{L})} \frac{d \tilde{L}}{\tilde{L}}=-\frac{\beta(1+\theta)}{[\rho(1+\theta)-\beta](\rho-\beta)} d \rho+\frac{\rho}{\rho(1+\theta)-\beta} d \theta \\
& \frac{1}{(1-\tilde{L})} \frac{d \tilde{L}}{\tilde{L}}=\frac{\beta(1+\theta)}{[\kappa(1+\theta)+\beta](\kappa+\beta)} d \kappa+\frac{\kappa}{\kappa(1+\theta)+\beta} d \theta
\end{aligned}
$$

From equations (26), (29a) and (29b) we can establish the following proposition, where for convenience we assume that $\theta>0$, so that the reference consumption level yields positive utility.

Proposition 3: Assume that preferences are described by the conventional constant elasticity utility function (27). Then:

(i) An external consumption reference point has no effect on real activity.

(ii) Increasing the importance of the internally formed reference consumption level - whether backward-looking or forward-looking - increases long-run employment, and thus, capital, output, and consumption. 
(iii) The long-run effect of an increase in $\rho$, the weight on near-future consumption in the anticipated future consumption reference level is contractionary.

(iv) The long-run effect of an increase in $\kappa$, the weight on the immediate past consumption in the habit-base consumption reference level is expansionary.

The contrast between (iii) and (iv) arises because of the constraint $\rho>\beta$ and that with looking-forward reference point the long-run shadow value of anticipations, $\tilde{\mu}<0$ is negative. The intuition underlying (ii) is similar to that discussed by Turnovsky and Monteiro (2007, Sec. 5) in their extended discussion of backward-looking habits and elastic labor supply.

\section{Conclusions}

It has long been argued that the anticipations of some future event will impact current consumption, in advance of the occurrence of the event itself. The current paper has introduced this idea of anticipated pleasure into an otherwise standard Ramsey growth model, by assuming that in addition to his own current consumption, an agent's current utility depends upon a reference consumption level that is based on a discounted measure of expected future consumptions. Following the "habit formation" literature, we have considered two alternative specifications of the anticipated future consumption levels: an external index and an internal index. We have then proceeded to analyze the macrodynamic equilibrium, comparing it to both the standard Ramsey model, while also contrasting it with the conventional model of habit formation. The approach we have taken is to combine the theoretical analysis with numerical simulations based on the calibration of a plausible macroeconomic growth model.

We have drawn three main sets of theoretical conclusions: First, the introduction of an external forward-looking consumption reference will decrease the rate of convergence if expectations of future consumption decrease the marginal utility of current consumption. This in turn will imply that capital will be accumulated less rapidly to make up the current shortfall in the capital stock and that current consumption will react slower to the accumulation of capital. Second, 
if labor is supplied inelastically, then steady-state consumption and output are identical to that of conventional Ramsey model, independently of how the consumption reference point is defined, and the direction of its impact on utility. Third, if labor is supplied elastically, the long-run employment, capital, output, and consumption all increase with the positive impact of the consumption reference on utility. On the other hand, increasing the speed of adjustment of the reference benchmark has a contractionary (expansionary) effect on steady state values of employment, capital, output and consumption if the reference stock is forward-looking (backward-looking).

Numerical simulations have supplemented these theoretical findings with important quantitative insights. One striking finding is the sharp contrast in the impact a forward-looking internal consumption reference has on the transitional adjustments of key variables, depending upon whether its effect on utility is positive or negative. This is contrary to the more common case of the backward-looking internal reference where the effects are much less sensitive to this issue. The difference reflects the fact that the forward-looking index can jump on impact, whereas the backward-looking index is constrained to gradual adjustments.

Finally, we should remind ourselves that the introduction of anticipated consumption into utility raises a range of issues addressed by psychologists having to do with emotions, the processing of information, and the key role of uncertainty ; see Elster and Loewenstein (1992). A desirable next step would be to begin to incorporate some of these elements into macrodynamic models such as this. 
Table 1:

A. Benchmark parameters

\begin{tabular}{|l|c|}
\hline Production parameters & $\alpha=1, \sigma=0.65, \delta=0.065$ \\
\hline Preference parameters & $\beta=0.04, \varepsilon=2.5, \rho=0.20, \theta=0.5,-0.5$ \\
\hline
\end{tabular}

\section{B. Eigenvalues}

\begin{tabular}{|l|c|c|}
\hline & $\theta=0.5$ & $\theta=-0.5$ \\
\hline Ramsey & -0.0626 & -0.0626 \\
\hline External -anticipation & -0.0548 & -0.0729 \\
\hline External - habit & $-0.0506,-0.2652$ & $-0.1057 \pm 0.0188 i(r=0.1074)$ \\
\hline Internal- anticipation & $-0.0502,-0.2518$ & $-0.0661 \pm 0.0401 i(r=0.0773)$ \\
\hline Internal - habit & $-0.0514,-0.2853$ & $-0.0936 \pm 0.0290 i(r=0.0980)$ \\
\hline
\end{tabular}


Table 2: Short-run and long-run responses to increase in $\alpha$ from 1 to 1.2

\begin{tabular}{|c|c|c|c|c|c|c|c|c|}
\hline & $\dot{K}(0)$ & $\tilde{K}$ & $A(0)$ & $\tilde{A}$ & $\dot{H}(0)$ & $\tilde{H}$ & $C(0)$ & $\overline{\tilde{C}}$ \\
\hline Base & $\mathbf{0}$ & 6.374 & 1.498 & 1.498 & $\mathbf{0}$ & 1.498 & 1.498 & 1.498 \\
\hline Ramsey & 0.129 & 8.438 & & & & & 1.751 & 1.983 \\
\hline $\begin{array}{ll}\text { External: } \quad \mathrm{R}=\mathrm{A} \\
\theta=0.5 \\
\theta=-0.5\end{array}$ & $\begin{array}{l}0.093 \\
0.130\end{array}$ & $\begin{array}{l}8.438 \\
8.438\end{array}$ & $\begin{array}{l}1.829 \\
1.812\end{array}$ & $\begin{array}{l}1.983 \\
1.983\end{array}$ & & & $\begin{array}{l}1.787 \\
1.750\end{array}$ & $\begin{array}{l}1.983 \\
1.983\end{array}$ \\
\hline $\begin{array}{l}\text { External: } \quad \mathrm{R}=\mathrm{H} \\
\theta=0.5 \\
\theta=-0.5\end{array}$ & $\begin{array}{l}0.056 \\
0.196\end{array}$ & $\begin{array}{l}8.438 \\
8.438\end{array}$ & & & $\begin{array}{l}0.065 \\
0.037\end{array}$ & $\begin{array}{l}1.983 \\
1.983\end{array}$ & $\begin{array}{l}1.824 \\
1.684\end{array}$ & $\begin{array}{l}1.983 \\
1.983\end{array}$ \\
\hline $\begin{array}{ll}\text { Internal: } \quad \mathrm{R}=\mathrm{A} \\
\theta=0.5 \\
\theta=-0.5\end{array}$ & $\begin{array}{l}0.466 \\
-0.343\end{array}$ & $\begin{array}{l}8.438 \\
8.438 \\
\end{array}$ & $\begin{array}{l}1.731 \\
2.025\end{array}$ & $\begin{array}{l}1.983 \\
1.983 \\
\end{array}$ & \multicolumn{2}{|c|}{ NA } & $\begin{array}{l}1.414 \\
2.223\end{array}$ & $\begin{array}{l}1.983 \\
1.983\end{array}$ \\
\hline $\begin{array}{ll}\text { Internal: } \quad \mathrm{R}=\mathrm{H} \\
\theta=0.5 \\
\theta=-0.5\end{array}$ & $\begin{array}{l}0.045 \\
0.201\end{array}$ & $\begin{array}{c}8.438 \\
8.438\end{array}$ & \multicolumn{2}{|c|}{$\mathbf{T}$} & $\begin{array}{l}0.067 \\
0.036\end{array}$ & $\begin{array}{l}1.983 \\
1.983\end{array}$ & $\begin{array}{l}1.835 \\
1.679\end{array}$ & $\begin{array}{l}1.983 \\
1.983\end{array}$ \\
\hline
\end{tabular}




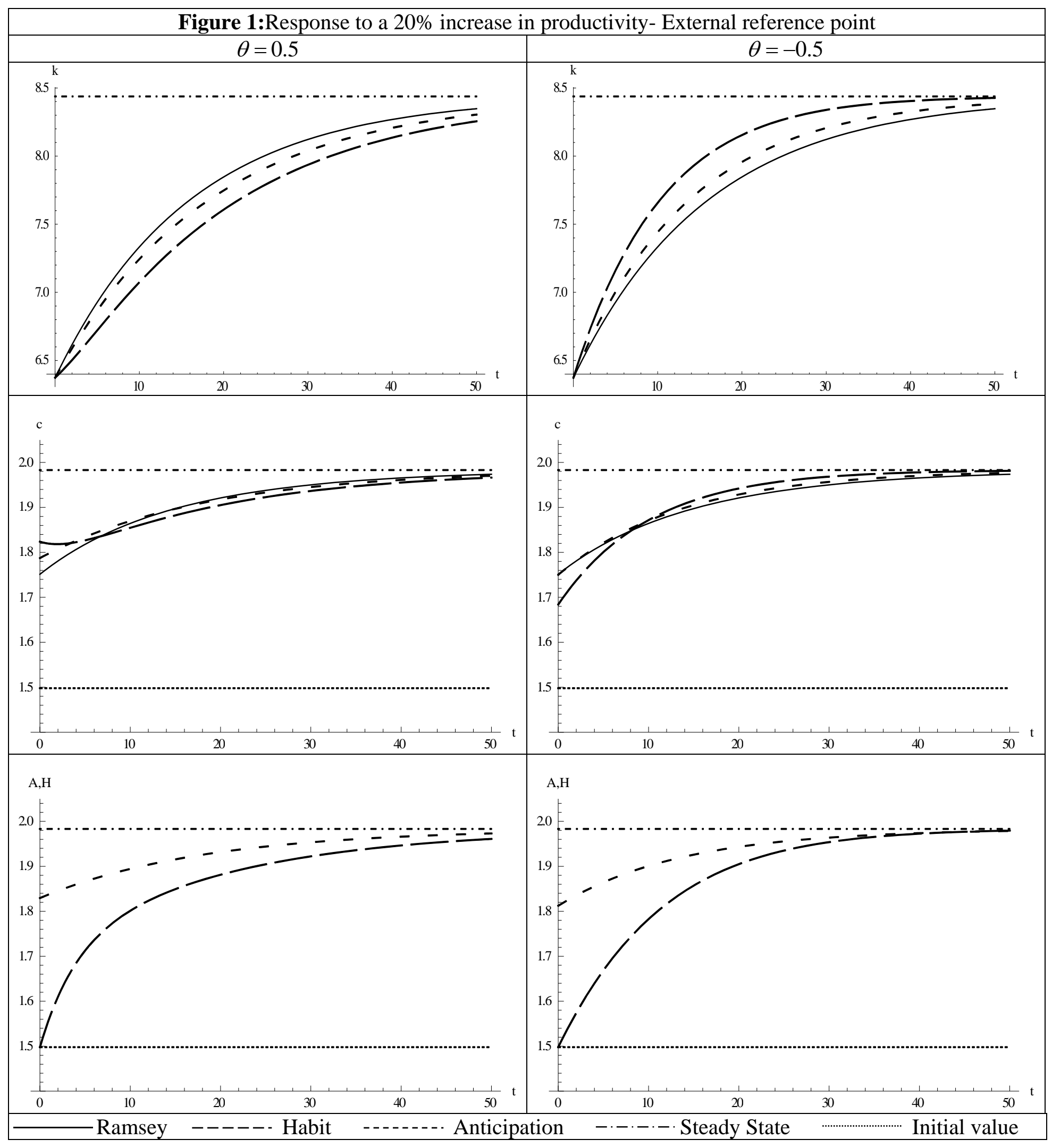




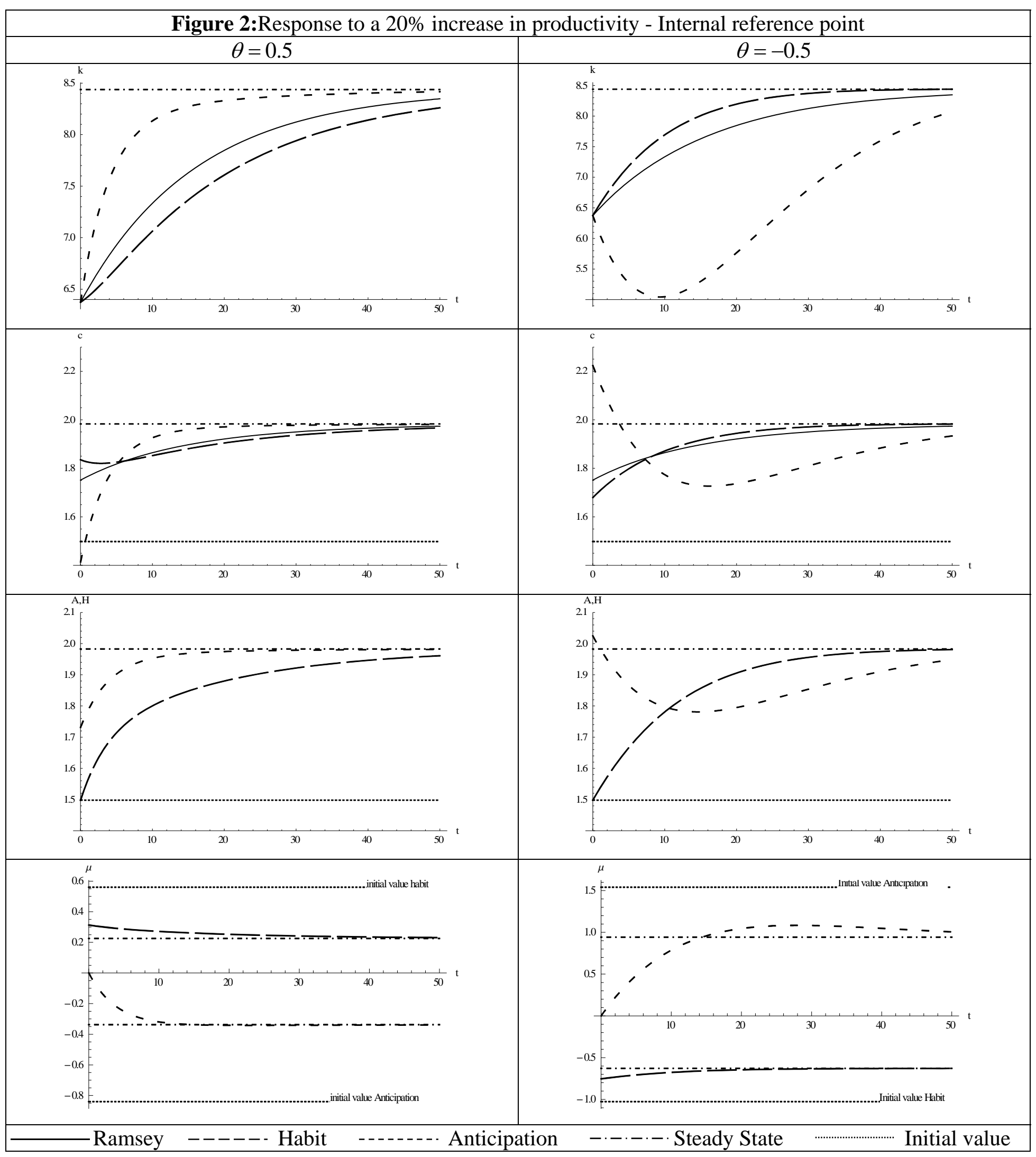




\section{Appendix}

\section{A.1 Properties of characteristic equation (12)}

The characteristic equation (12) can be written as:

$$
f(v) \equiv v^{3}-\left[\beta+\rho\left(1-C_{A}\right)\right] v^{2}+\left[\beta \rho\left(1-C_{A}\right)-\lambda C_{\lambda} F_{K K}\right] v+\rho \lambda C_{\lambda} F_{K K}=0
$$

Since the product of the three eigenvalues $v_{1} v_{2} v_{3}=\rho \lambda C_{\lambda} F_{K K}>0$, we know that there are either one

or three positive roots. By Descartes rule of signs we immediate see from (A.1) irrespective of whether $1_{<}^{>} C_{A}$, (A.1) involves exactly two sign changes and therefore has exactly one negative (stable) root $v_{1}<0$, and two positive roots, $v_{3}>v_{2}>0$. In order for the transversality condition to be met we require $2 v_{i}<\beta$, which clearly holds for the stable root. Examining (A.1) we can show (i) $f(0)>0$, (ii) $f^{\prime}\left(\beta / 2<0\right.$, which ensures that $2 v_{3}>\beta$, so that the largest root violates the transversality condition. A sufficient condition that $2 v_{2}>\beta$ is that $C_{A}<(2 \rho-\beta) / 2 \rho$, although weaker conditions can also be found.

The corresponding characteristic equation in the absence of anticipations is

$$
g(v) \equiv v^{2}-\beta v-\lambda C_{\lambda} F_{K K}=0
$$

Denote the stable root in the latter case as $v^{*}<0$, so that $g\left(v^{*}\right)=0$ and $v^{*}<\beta$. Writing (A.1) in the form

$$
f(v)=(v-\rho) g(v)+\rho C_{A} v(v-\beta)
$$

we obtain

$$
f\left(v^{*}\right)=\rho C_{A} v^{*}\left(v^{*}-\beta\right)
$$

so that $\operatorname{sgn}\left(f\left(v^{*}\right)=\operatorname{sgn}\left(C_{A}\right)\right.$ Thus:

(i) If $U_{C A}>0$ so that $C_{A}>0$ then $f\left(v^{*}\right)>0$ implying $v_{1}<v^{*}<0$

(ii) If $U_{C A}<0$ so that $C_{A}<0$ then $f\left(v^{*}\right)<0$ implying $v^{*}<v_{1}<0$ 


\section{A.2 Equilibrium dynamics with externally generated backward-looking reference}

The equilibrium dynamics in this case is described by the set of equations

$$
\begin{aligned}
& \dot{K}(t)=F(K, \bar{L})-C(\lambda, H)-\delta K \\
& \dot{\lambda}(t)=\lambda\left[\beta+\delta-F_{K}(K, \bar{L})\right] \\
& \dot{H}(t)=\kappa[C(\lambda, H)-H]
\end{aligned}
$$

where $H(t)$ is now backward-looking. The transversality conditions and steady state remain unchanged, while the linearized dynamics are now described by

$$
\left(\begin{array}{c}
\dot{K}(t) \\
\dot{\lambda}(t) \\
\dot{H}(t)
\end{array}\right)=\left(\begin{array}{ccc}
\beta & -C_{\lambda} & -C_{H} \\
-\tilde{\lambda} F_{K K} & 0 & 0 \\
0 & \kappa C_{\lambda} & -\kappa\left(1-C_{H}\right)
\end{array}\right)\left(\begin{array}{c}
K(t)-\tilde{K} \\
\lambda(t)-\tilde{\lambda} \\
H(t)-\tilde{H}
\end{array}\right)
$$

so that the characteristic equation is now modified to

$$
f(v) \equiv v^{3}-\left[\beta-\kappa\left(1-C_{H}\right)\right] v^{2}-\left[\beta \kappa\left(1-C_{H}\right)+\lambda C_{\lambda} F_{K K}\right] v-\kappa \lambda C_{\lambda} F_{K K}=0
$$

The product of the three eigenvalues is positive, implying that there are either two negative and one positive root, or that all three roots are positive. Descartes rule of signs indicates that irrespective of whether $1_{<}^{>} C_{H}$, there is only one sign change and therefore only one positive root.

The stable adjustment path is therefore given by the two dimensional manifold

$$
\begin{aligned}
& K(t)=\tilde{K}+B_{1} e^{v_{1} t}+B_{2} e^{v_{2} t} \\
& \lambda(t)=\tilde{\lambda}+B_{1} \omega_{21} e^{v_{1} t}+B_{2} \omega_{22} e^{v_{2} t} \\
& H(t)=\tilde{H}+B_{1} \omega_{31} e^{v_{1} t}+B_{2} \omega_{32} e^{v_{2} t}
\end{aligned}
$$

where now $v_{1}<0, v_{2}<0$ are the stable eigenvalues and $\omega_{i j}$ are elements of the corresponding normalized eigenvectors, defined by 


$$
\left(\begin{array}{ccc}
F_{K} & -C_{\lambda} & -C_{H} \\
-\tilde{\lambda} F_{K K} & 0 & 0 \\
0 & \kappa C_{\lambda} & -\kappa\left(1-C_{H}\right)
\end{array}\right)\left(\begin{array}{c}
1 \\
\omega_{2 i} \\
\omega_{3 i}
\end{array}\right)=v_{i}\left(\begin{array}{c}
1 \\
\omega_{2 i} \\
\omega_{3 i}
\end{array}\right) \quad i=1,2
$$

The arbitrary constants $B_{1}, B_{2}$ are determined by the initial on the sluggish variables $H, K$, namely

$$
\begin{aligned}
& B_{1}+B_{2}=\tilde{K}-K_{0} \\
& B_{1} \omega_{31}+B_{2} \omega_{32}=H_{0}-\tilde{H}
\end{aligned}
$$

and depend upon the underlying structural change.

\section{A.3 Equilibrium dynamics with internally generated backward-looking reference}

This is the conventional case discussed by Carroll et al (1997), Alvarez-Cuadrado et al (2004), and many others. The underlying dynamics are

$$
\begin{aligned}
& \dot{K}(t)=F(K)-C(\lambda, H, \mu)-\delta K \\
& \dot{\mu}=(\beta+\kappa) \mu-U_{H}(C(H, \lambda, \mu), H) \\
& \dot{\lambda}=\lambda\left[\beta-F_{K}(K)\right] \\
& \dot{H}=\kappa(C(H, \lambda, \mu)-H)
\end{aligned}
$$

Steady state, obtained when $\dot{K}=\dot{H}=\dot{\lambda}=\dot{\mu}=0$, is described by

$$
\begin{aligned}
& F(\tilde{K})=\tilde{C}+\delta \tilde{K} \\
& F_{K}(\tilde{K})=\beta+\delta \\
& \tilde{H}=\tilde{C} \\
& U_{C}(\tilde{C}, \tilde{H})=\tilde{\lambda}-\kappa \tilde{\mu} \\
& (\beta+\kappa) \tilde{\mu}-U_{H}(\tilde{C}, \tilde{H})=0
\end{aligned}
$$


To obtain the transitional dynamics we linearize (A.9) around (A.10):

$$
\left(\begin{array}{c}
\dot{K} \\
\dot{\mu} \\
\dot{\lambda} \\
\dot{H}
\end{array}\right)=\left(\begin{array}{cccc}
\beta & -C_{\mu} & -C_{\lambda} & -C_{H} \\
0 & (\beta+\kappa)-U_{H C} C_{\mu} & -U_{H C} C_{\lambda} & -\left[U_{H H}+U_{H C} C_{A}\right] \\
-F_{K K} & 0 & 0 & 0 \\
0 & \kappa C_{\mu} & \kappa C_{\lambda} & -\kappa\left(1-C_{H}\right)
\end{array}\right)\left(\begin{array}{c}
K-\tilde{K} \\
\mu-\tilde{\mu} \\
\lambda-\tilde{\lambda} \\
H-\tilde{H}
\end{array}\right)
$$

The characteristic equation is now given by,

$$
D \equiv v^{4}-2 \beta v^{3}+\theta_{2} v^{2}+\theta_{3} v+\frac{F_{K K}}{U_{C C}} \kappa(\kappa+\beta)=0
$$

where

$$
\begin{aligned}
& \theta_{2} \equiv \beta^{2}+\left[\kappa C_{\mu} U-\kappa\left(1-C_{H}\right)\left[\beta+\kappa\left(1-C_{H}\right)\right]\right]-\frac{F_{K K}}{U_{C C}} \equiv \Omega+\beta^{2} \\
& \theta_{3} \equiv \beta\left[\kappa\left(1-C_{H}\right)\left[\beta+\kappa\left(1-C_{H}\right)\right]-\kappa C_{\mu} U\right]+\beta \frac{F_{K K}}{U_{C C}} \equiv-\beta \Omega
\end{aligned}
$$

Since $\left(F_{K K} / U_{C C}\right) \kappa(\kappa+\beta)>0$, and $\theta_{3}>0$, (A.12) implies that there are two stable and two unstable roots so that the equilibrium dynamics are a well defined saddlepoint. The solution is of same form as for the internally generated expectational reference, except that the sluggish variables are now $K, H$, namely

$$
\begin{aligned}
& K(t)-\tilde{K}=B_{1} e^{v_{1} t}+B_{2} e^{\nu_{2} t} \\
& \mu(t)-\tilde{\mu}=B_{1} \omega_{21} e^{\nu_{1} t}+B_{2} \omega_{22} e^{\nu_{2} t} \\
& \lambda(t)-\tilde{\lambda}=B_{1} \omega_{31} e^{v_{1} t}+B_{2} \omega_{32} e^{v_{2} t} \\
& H(t)-\tilde{H}=B_{1} \omega_{41} e^{v_{1} t}+B_{2} \omega_{42} e^{v_{2} t}
\end{aligned}
$$

where $B_{1}, B_{2}$ are arbitrary, $K(0)=K_{0}, H(0)=H_{0}$, and the vector $\left(1, \omega_{2 i}, \omega_{3 i}, \omega_{4 i}\right), i=1,2$ is the normalized eigenvector associated with the stable eigenvalue $v_{i}$. That is $\left(1, \omega_{2 i}, \omega_{3 i}, \omega_{4 i}\right)$ satisfies 


$$
\left(\begin{array}{cccc}
\beta-v_{i} & -C_{\mu} & -C_{\lambda} & -C_{A} \\
0 & (\beta+\kappa)-U_{H C} C_{\mu}-v_{i} & -U_{H C} C_{\lambda} & -\left[U_{H H}+U_{H C} C_{H}\right] \\
-F_{K K} & 0 & -v_{i} & 0 \\
0 & \kappa C_{\mu} & \kappa C_{\lambda} & -\kappa\left(1-C_{H}\right)-v_{i}
\end{array}\right)\left(\begin{array}{c}
1 \\
\omega_{2 i} \\
\omega_{3 i} \\
\omega_{4 i}
\end{array}\right)=0
$$

The constants $B_{1}, B_{2}$ are obtained from the initial conditions

$$
\begin{aligned}
& B+B_{2}=K_{0}-\tilde{K} \\
& \omega_{41} B_{1}+\omega_{42} B_{2}=H_{0}-\tilde{H}
\end{aligned}
$$




\section{References}

Abel, A. (1990), Asset prices under habit formation and catching up with the Joneses, American Economic Review 80, 38-42.

Alonso-Carrera J., J. Caballé, and X. Raurich (2005), Growth, habit formation and catching-up with the Joneses, European Economic Review 49, 1665-1691.

Alvarez-Cuadrado, F., G. Monteiro, and S.J. Turnovsky (2004), Habit formation, catching up with the Joneses, and non-scale growth, Journal of Economic Growth, 9, 47-80.

Boyer, M. (1978), A habit forming optimal growth model, International Economic Review 19, 585609.

Braun, P.A., G.M. Constantinides and W.E. Ferson (1993), Time nonseparability of aggregate consumption: international evidence, European Economic Review, 37, 897-920

Campbell, J.Y., and J.N. Cochrane (1999), By force of habit: A consumption-based explanation of aggregate stock behavior, Journal of Political Economy, 107, 205-251.

Caplin, A. and J. Leahy (2001), Psychological expected utility theory and anticipatory feelings, Quarterly Journal of Economics 116, 55-79.

Carroll, C.D, J. R. Overland, and D. N. Weil (1997), Comparison utility in a growth model, Journal of Economic Growth, 2, 339-367.

Carroll, C.D., J. R. Overland, and D.N. Weil (2000), Saving and growth with habit formation, American Economic Review 90, 341-355.

Constantinides, G.M. (1990), Habit formation: A resolution of the equity premium puzzle, Journal of Political Economy 98, 519-543.

Duesenberry, J. (1949), Income, Saving, and the Theory of Consumer Behavior, Harvard University Press, Cambridge MA.

Dupor, B. and W.F. Liu (2003), Jealousy and equilibrium overconsumption, American Economic Review 93, 423-428.

Dynan, K.E. (2000), Habit formation in consumer preferences: Evidence from panel data, American Economic Review 90, 391-406. 
Elster, J. and G. Loewenstein (1992), Utility from memory and anticipation, in G. Loewensten and J. Elster (eds.), Choice over Time, Russell Sage Foundation, NewYork, 213-234.

Faria, J.R. and P. McAdam (2013), Anticipation of future consumption: A monetary perspective, Journal of Money, Credit, and Banking 45, 423-447 .

Fearson, W.E. and G.M. Constantinides (1991), Habit persistence and durability in aggregate consumption: Empirical tests, Journal of Financial Economics 29, 199-240.

Fuhrer, J.C. (2000), Habit formation in consumption and its implications for monetary-policy models, American Economic Review 90, 367-390.

Gali, J. (1994), Keeping up with the Joneses: Consumption externalities, portfolio choice, and asset prices, Journal of Money, Credit, and Banking, 26, 1-8.

Hestenes, M.R. (1966), Calculus of Variations and Optimal Control Theory, Wiley, New York.

Houthakker, H.S. and L.D. Taylor (1966), Consumer Demand in the United States, Harvard University Press, Cambridge MA.

Jevons, W.S. (1871), Essays on Economics, Macmillan, London.

Kamien, M.I. and N.L. Schwartz (1971), Sufficient conditions in optimal control theory, Journal of Economic Theory 3, 207-214.

Köszegi, B, and M. Rabin (2006), A model of reference-dependent preferences, Quarterly Journal of Economics 121, 1133-1165.

Kőszegi, B, and M. Rabin (2007), Reference-dependent risk attitudes, American Economic Review 97, 1047-1073.

Kőszegi, B. (2010), Utility from anticipation and personal equilibrium, Economic Theory 44, 415444.

Kurz, M. (1968), Optimal economic growth and wealth effects, International Economic Review 9, 348-357.

Kuznitz, A., S. Kandel, V. Fos (2008), A portfolio choice model with utility from anticipation of future consumption and stock market mean reversion, European Economic Review 52, 13381352. 
Leonard, D. and N.V. Long (1992), Optimal Control Theory and Static Optimization in Economics, Cambridge University Press, Cambridge UK.

Liu, W.F. and S.J. Turnovsky (2005), Consumption externalities, production externalities, and longrun macroeconomic efficiency, Journal of Public Economics, 89, 1097-1029.

Ljungqvist, L. and H. Uhlig (2000), Tax policy and aggregate demand management under catching up with the Joneses, American Economic Review 90, 356-366.

Lowenstein, G. (1987), Anticipation and the valuation of delayed consumption, Economic Journal $97,666-684$.

Mansoorian, A. (1998), Habits and durability in consumption and the dynamics of the current account, Journal of International Economics 44, 69-92.

Marshall, A. (1891), Principles of Economics $2^{\text {nd }}$ ed. Macmillan, London.

Osborn, D.R. (1988), Seasonality and habit persistence in a life cycle model of consumption, Journal of Applied Econometrics, 3, 255-266.

Rubin, D.C., S.E. Wetzler, and R.D. Nebes (1986), Autobiographical memories across the adult lifespan, in D.C. Rubin (ed.) Autobiographical Memory, Kluwer, Dordrecht, the Netherlands.

Ryder, H.E. and G.M. Heal (1973), Optimal growth with intertemporally dependent preferences, Review of Economic Studies, 40, 1-31.

Taylor, L.D. and H.S. Houthakker (2010), Consumer Demand in the United States, Third Edition, Springer, New York and Heidelberg.

Turnovsky S.J. and G Monteiro (2007), Consumption externalities, production externalities, and efficient capital accumulation under time non-separable preferences, European Economic Review 51, 479-504.

Veblen, T. (1912), The Theory of the Leisure Class, Macmillan, New York.

Wendner, R. (2011), Will the consumption externalities' effects in the Ramsey model please stand up? Economics Letters 111, 210-212.

Zou, H.F. (1999), The spirit of capitalism and long-run growth, European Journal of Political Economy 10, 279-293. 